\title{
Omega-3 polyunsaturated fatty acids supplementation improve clinical symptoms in patients with covid-19: A randomized clinical trial
}

\author{
Mohsen Sedighiyan ${ }^{1}$, Hamed Abdollahi ${ }^{1}$, Elmira Karimi ${ }^{2}$, Mostafa Badeli ${ }^{1}$, Reza Erfanian ${ }^{1}$, \\ Shima Raeesi ${ }^{1}$, Rezvan Hashemi ${ }^{1}$, Zahra Vahabi ${ }^{1}$, Behzad Asanjarani ${ }^{1}$, and Mina \\ ABDOLAHI ${ }^{1}$
}

${ }^{1}$ Affiliation not available
${ }^{2}$ Tehran University of Medical Sciences

January 13, 2021

\begin{abstract}
Objective: We hypothesized that omega-3 fatty acids would be an appropriate adjunct therapy for alleviating the inflammatory response and clinical manifestation in hospitalized patients with covid-19 disease. Methods: This was a single-blind randomized controlled trial in Amir-Alam hospital in Tehran. Thirty adult men and women diagnosed with covid-19 were allocated to either control group (receiving Hydroxychloroquine) or intervention group (receiving Hydroxychloroquine plus 2 grams of DHA+EPA) for 2 weeks. Primary outcome of the intervention including CRP, ESR as well as clinical symptoms including body pain, fatigue, appetite and olfactory and secondary outcomes including liver enzymes were determined at the baseline and after omega-3 supplementation. Clinical signs were measured using self-reported questionnaires. There were commercial kits for determination of CRP and liver enzymes concentrations in the serum of patients. For determination of ESR automated hematology analyzer was applied. Results: In comparison to control group, patients receiving omega-3 indicated favorable changes in all clinical symptoms except for olfactory $((\mathrm{p}<0.001$ for body pain and fatigue, $\mathrm{p}=0.03$ for appetite and $\mathrm{p}=0.21$ for olfactory). Reducing effects of omega-3 supplementation compared to control group were also observed in the levels of ESR and CRP after treatment $(\mathrm{p}<0.001$ for CRP and $\mathrm{p}=0.02$ for ESR). However, no between group differences in the liver enzymes serum concentrations were observed after supplementation $(\mathrm{p}>0.05)$. Conclusion: Current observations are very promising and indicate that supplementation with moderate dosages of omega-3 fatty acids may be beneficial in the management of inflammationmediated clinical symptoms in covid-19 patients. Key words: Covid-19, omega-3, inflammation, clinical symptoms
\end{abstract}

\section{Hosted file}

Manuscript.omega-3(1)-final.pdf available at https://authorea.com/users/389148/articles/ 503775-omega-3-polyunsaturated-fatty-acids-supplementation-improve-clinical-symptoms-inpatients-with-covid-19-a-randomized-clinical-trial 